\title{
Zero Modes and Global Antiferromagnetism in Strained Graphene
}

\author{
Bitan Roy \\ National High Magnetic Field Laboratory and Department of Physics, \\ Florida State University, Tallahassee, Florida 32306, USA \\ and Condensed Matter Theory Center, Department of Physics, University of Maryland, \\ College Park, Maryland 20742, USA
}

Fakher F. Assaad

Institut für Theoretische Physik und Astrophysik, Universität Würzburg, Am Hubland, D-97074 Würzburg, Germany and Max-Planck-Institut für Physik komplexer Systeme, Nöthnitzer Strasse 38, 01187 Dresden, Germany

Igor F. Herbut

Max-Planck-Institut für Physik komplexer Systeme, Nöthnitzer Strasse 38, 01187 Dresden, Germany and Department of Physics, Simon Fraser University, Burnaby, British Columbia, Canada V5A IS6 (Received 28 January 2014; revised manuscript received 23 March 2014; published 30 May 2014)

A novel magnetic ground state is reported for the Hubbard Hamiltonian in strained graphene. When the chemical potential lies close to the Dirac point, the ground state exhibits locally both the Néel and ferromagnetic orders, even for weak Hubbard interaction. Whereas the Néel order parameter remains of the same sign in the entire system, the magnetization at the boundary takes the opposite sign from the bulk. The total magnetization vanishes this way, and the magnetic ground state is globally only an antiferromagnet. This peculiar ordering stems from the nature of the strain-induced single-particle zero-energy states, which have support on one sublattice of the honeycomb lattice in the bulk, and on the other sublattice near the boundary of a finite system. We support our claim with the self-consistent numerical calculation of the order parameters, as well as by the Monte Carlo simulations of the Hubbard model in both uniformly and nonuniformly strained honeycomb lattice. The present result is contrasted with the magnetic ground state of the same Hubbard model in the presence of a true magnetic field (and for vanishing Zeeman coupling), which is exclusively Néel ordered, with zero local magnetization everywhere in the system.

DOI: 10.1103/PhysRevX.4.021042

\section{INTRODUCTION}

Despite the manifest stability of the Dirac fermions in graphene against the effects of Coulomb interaction [1], the nature of the possible broken symmetry phases at strong coupling continues to be an issue of fundamental importance [2-13]. The minimal on-site Hubbard interaction, for example, when sufficiently strong, is believed to produce the antiferromagnetic Néel ground state [2,3,6,7,14-17]. The universality class of the semimetal-to-Néel insulator quantum phase transition can be captured by the effective Gross-Neveu-Yukawa field theory [18-20] and studied systematically near the upper critical (spatial) dimension of three. Recent quantum Monte Carlo simulations of the

Published by the American Physical Society under the terms of the Creative Commons Attribution 3.0 License. Further distribution of this work must maintain attribution to the author $(s)$ and the published article's title, journal citation, and DOI.
Subject Areas: Condensed Matter Physics, Graphene, Strongly Correlated Materials

Hubbard model in a half-filled honeycomb lattice suggest a direct transition from the Dirac semimetal to the Néel state, with the critical exponents in reasonable agreement with the field-theoretic predictions $[15,16]$. The correlated phases of graphene, Néel state included, unfortunately may be lying at too strong a coupling to be realized in graphene in its pristine state, even when placed in vacuum [21].

The deformability of the graphene membrane may facilitate a different path towards the realization of some of the symmetry-broken phases at weaker couplings [22]. Arguments along this line and in favor of the topological quantum anomalous (spin) Hall insulator at weak finiterange repulsion [22-24], or of unconventional superconductors[25] at weak attraction, have recently been put forward. The physical reason behind this electromechanical phenomenon is the generic appearance of the singleparticle zero-energy states in graphene under strain. Upon bulging a graphene flake, the quasirelativistic lowenergy electronic degrees of freedom couple to a finite, time-reversal-symmetric, magneticlike field [26]. Such an, 
and not necessarily uniform, axial magnetic field, similarly to the true magnetic field, falls under the jurisdiction of index theorems [27], and as such brings a finite number of states close to the Dirac point. This creates an ideal situation for the fermions to form various particle-hole or particle-particle condensates at weak interactions. The single-particle states at zero energy, responsible for the weak-coupling instabilities, however, are special in the axial case [22]: normalizability forces them to live exclusively on one of the two sublattices of the honeycomb lattice. The remaining states in the zero-energy subspace, which would be discarded from the spectrum as nonnormalizable in an infinite system, are found near the boundary in a finite system, and are living on the opposite sublattice.

Because of this inextricable correlation between the realspace and sublattice degrees of freedom within the zeroenergy subspace, the ground state of the Hubbard model in strained graphene is nontrivial. While it seems natural to expect that a short-range interaction such as Hubbard's would lead to a spin-polarized "Hund" ferromagnetic state in the presence of a flatband in strained graphene, we show here, first via a self-consistent numerical calculation, that the ground state of the Hubbard model in this system is more interesting: while it locally displays the expected ferromagnetic ordering, the sign of the magnetization varies in precisely such a way so that the total space-integrated magnetization in fact vanishes. Since the magnetization is tied to the zero modes, however, its support, as well as its sign, also switches between the two sublattices when traversing the system from its bulk to the boundary, so that the Néel order parameter is also finite, and of the same sign everywhere. The appearance of this unusual magnetic ordering is also supported by a quantum Monte Carlo calculation on the Hubbard model on a half-filled strained honeycomb lattice. We call this unconventional magnetic ordering the global (edge-compensated) antiferromagnet. Recent experimental progress in realizing the axial magnetic field in real and artificial graphene [28-30] offers hope for the detection of this unusual ground state.

The paper is organized as follows. In the next section, we propose a specific modulation of the nearest-neighbor hopping amplitude that introduces a finite axial magnetic field in a graphene flake. We also discuss and compare the zero modes in the presence of the strain-induced axial and true magnetic fields. In Sec. III, we introduce the on-site Hubbard interaction and discuss possible magnetic ground states in strained graphene. In Sec. IV, we present the selfconsistent Hatree solution for the magnetic ground state in the presence of (roughly) uniform and well-localized axial magnetic fields. We contrast our results with the magnetic ground state in graphene in a true (time-reversal symmetrybreaking) magnetic field in Sec. V. Quantum Monte Carlo simulation of the Hubbard model in strained graphene is presented in Sec. VI. We summarize our findings in Sec. VII.

\section{AXIAL MAGNETIC FIELD AND ZERO MODES}

Before delving into the effects of the electron-electron interactions, let us set the stage by reviewing the physics of zero modes in the continuum and on the lattice, and by introducing a specific realization of the axial magnetic fields on a finite honeycomb lattice. As is well known, the low-energy degrees of freedom in graphene may be collected into an eight-component Dirac spinor $\Psi=\left[\Psi_{\uparrow}, \Psi_{\downarrow}\right]^{\top}$, where $\Psi_{\sigma}^{\top}(\vec{q})=\left[u_{\sigma}(\vec{K}+\vec{q}), v_{\sigma}(\vec{K}+\vec{q})\right.$, $\left.u_{\sigma}(-\vec{K}+\vec{q}), v_{\sigma}(-\vec{K}+\vec{q})\right]$, and $\sigma=\uparrow, \downarrow$ are the two projections of electron spin along the $z$ axis [6,7]. $u_{\sigma}$ is the fermionic annihilation operator on the $A$ sublattice, generated by the linear combination of basis vectors $\vec{a}_{1}=(\sqrt{3},-1) a, \vec{a}_{2}=(0,1) a$, where $a$ is the lattice spacing and $v_{\sigma}^{\dagger}$ is the fermionic creation operator on the $B$ sublattice, where $\vec{B}=\vec{A}+\vec{c}$, with $\vec{c}=(1 / \sqrt{3}, 1)(a / 2)$. Two inequivalent Dirac points, or valleys, may be chosen as at $\pm \vec{K}$, where $\vec{K}=(1,1 / \sqrt{3})(2 \pi / a \sqrt{3})$. When $|\vec{q}| \ll|\vec{K}|$, the noninteracting low-energy Hamiltonian with only the nearest-neighbor hopping takes the relativistically invariant form $H_{D}=\sigma_{0} \otimes i \gamma_{0} \gamma_{j} \hat{q}_{j}$, with the first matrix acting on spin, and the second on sublattice and valley indices.

The coupling of the time-reversal-symmetric axial magnetic field $b(\vec{x})$ to the low-energy Dirac fermions then reads as $[22,31-34]$

$$
H[\vec{a}]=\sigma_{0} \otimes i \gamma_{0} \gamma_{j}\left[q_{j}-i \gamma_{3} \gamma_{5} a_{j}(\vec{x})\right] \equiv M(\chi) H_{D} M(\chi),
$$

where $b(\vec{x})=\epsilon_{i j} \partial_{i} a_{j}$ and $M(\chi)=\exp \left[\left(\sigma_{0} \otimes \gamma_{0}\right) \chi(\vec{x})\right]$. The axial vector potential here is $a_{i}(\vec{x})=\epsilon_{i j} \partial_{j} \chi(\vec{x})$ and, therefore, $b(\vec{x})=\partial^{2} \chi(\vec{x})$. The mutually anticommuting four-dimensional $\gamma$ matrices may be represented as $\gamma_{0}=\sigma_{0} \otimes \sigma_{3}, \gamma_{1}=\sigma_{3} \otimes \sigma_{2}, \gamma_{2}=\sigma_{0} \otimes \sigma_{1}, \gamma_{3}=\sigma_{1} \otimes \sigma_{2}$, $\gamma_{5}=\sigma_{2} \otimes \sigma_{2} .\left(\sigma_{0}, \vec{\sigma}\right)$ are the usual two-dimensional Pauli matrices. In this representation, the time-reversal symmetry operator is (antilinear) $I_{K}=\sigma_{2} \otimes i \gamma_{1} \gamma_{5} K$, where $K$ is the complex conjugation [6,7].

A random distribution of the axial gauge field in graphene, for example, results from the presence of ripples, with the net axial flux as zero. If graphene is deliberately buckled, on the other hand, a finite total flux of the axial field may be introduced, which by index theorem would bring a finite number of states at zero energy. These special zero modes can be written as

$$
\Psi_{0, n}[\vec{a}](\vec{x}) \propto e^{-\chi(\vec{x})\left(\sigma_{0} \otimes \gamma_{0}\right)} \Psi_{0, n}[0](\vec{x}) .
$$

The number of zero-energy states labeled by index $n$ equals the total axial flux through the system. The matrix $\gamma_{0}$ in the exponent changes sign between two sublattices, whereas the function $\chi(\vec{x})$ is a monotonic function at a large distance $|\vec{x}|$ from the location of the axial flux. The normalizable zero-energy states, therefore, must reside only on one of the two sublattices, which we call sublattice $A$. On the finite 
honeycomb lattice, these are the bound states in the interior of the system, where the flux is located. The remaining non-normalizable zero-energy states, with the support on the sublattice $B$, on the other hand, in the continuum increase exponentially towards infinity. On a finite lattice, however, this is tantamount to their localization near the boundary of the system.

Equation (1) suggests an introduction of an axial magnetic field on a lattice via the following modification of the nearest-neighbor hopping amplitude $(t)$ :

$$
t_{i j} \rightarrow e^{\chi(i)} t e^{-\chi(j)}
$$

where $i \in A, j \in B$ [24,35]. Hereafter, we set $t=1$ for convenience. Let us define a quantity, say $\mathcal{R}$, which counts the minimal number of bonds required to reach a particular site in the system from the central hexagon [centered at $\left.\left(j_{x}, j_{y}\right)=(0,0)\right]$ in Fig. 1. For all of the six sites on the central hexagon of the system, $\mathcal{R}=0$, for example. We then assign the parameter $\chi(\mathcal{R})$, such that for all the sites with the same $\mathcal{R}, \chi(\mathcal{R})$ is the same, as shown in Fig. 1, and the hoppings between nearest-neighbor sites are modified according to Eq. (3). Otherwise, $\chi(\mathcal{R})$ increases monotonically from the center towards the boundary of the system. As a result, a finite axial magnetic field is introduced in the system. For example, if $\chi(\mathcal{R}) \sim \mathcal{R}^{2}$, the system experiences a roughly uniform axial field, whereas a bell-shaped localized axial flux around the center of the system can be realized by setting $\chi(\mathcal{R}) \sim \log \mathcal{R}$. This configuration of $\chi(\mathcal{R})$ is slightly different than in the previous work [24], with the advantage that the increase of the bandwidth in the presence of axial fields can be somewhat better controlled here. Upon introducing such modification in the nearestneighbor hopping amplitude, the strained honeycomb lattice, shown in Fig. 1, is invariant under a $C_{3}$ symmetry, and thus the applied strain in our system is triaxial.

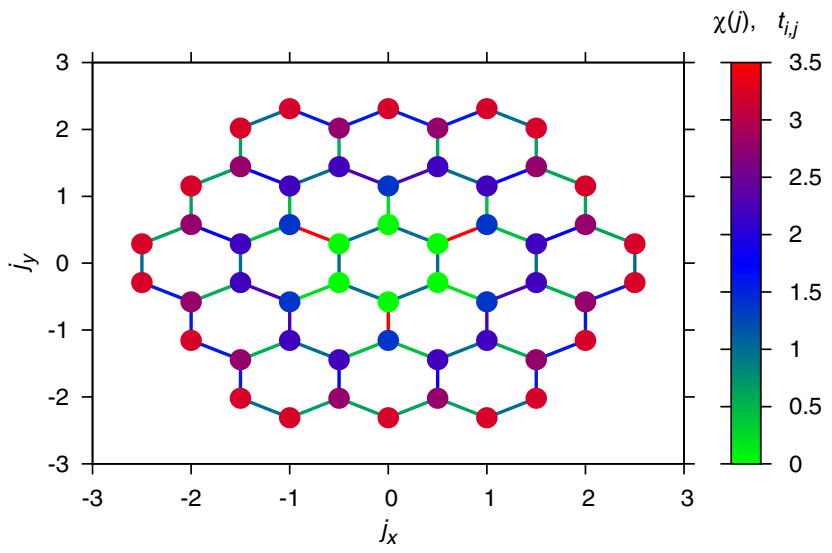

FIG. 1. A realization of strained honeycomb lattice that yields a finite axial magnetic field. Colors at each site correspond to the parameter $\chi$ in Eq. (3), which defines the modification of the hopping amplitude along each bond.
In a finite strained honeycomb lattice there are, of course, no states at precise zero energy. Nevertheless, irrespective of the spatial profile of the axial magnetic field, there are always a finite number of states in close vicinity to the zero energy [24] that bear the signature of the axial magnetic field. Let us consider two such states $| \pm \delta\rangle$, with the energies $\pm \delta$, where $|\delta| \sim 0$. The symmetric $|S\rangle$ and the antisymmetric $|A S\rangle$ combinations of these two states live on the $A$ and $B$ sublattices, respectively. In the presence of axial magnetic fields, $|S\rangle$ lives inside the bulk, as shown in Fig. 2 (top) and corresponds to the normalizable zero-energy states in Eq. (2). The $|A S\rangle$ lives near the boundary of a finite system, as shown in Fig. 2 (bottom), and is to be identified as the non-normalizable state in the continuum description. Hence, the bulk and the boundary of a finite graphene system become inequivalent in their sublattice structure in the presence of axial magnetic fields.
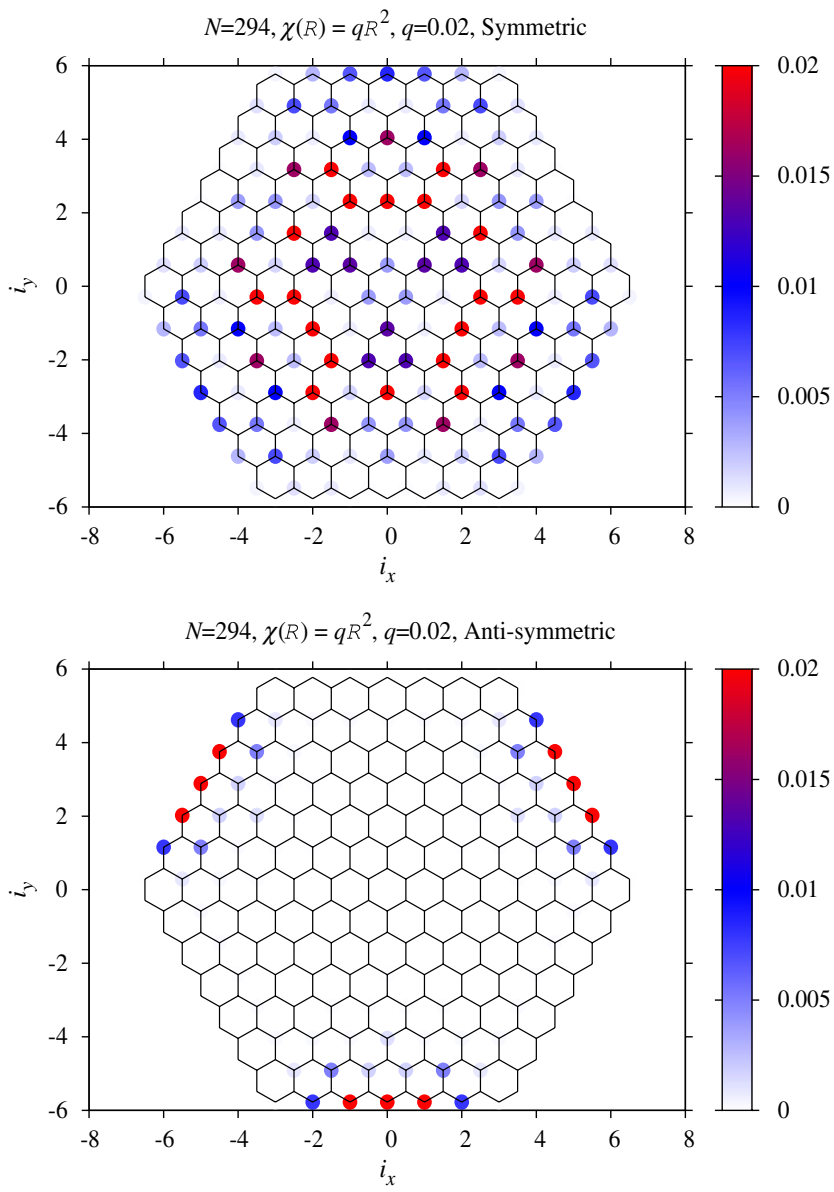

FIG. 2. Top: Spatial distribution of the symmetric combination $(|S\rangle)$ of the wave functions $| \pm \delta\rangle$, living on the $A$ sublattice, in a system of 294 sites or $r_{\max }=7$. Bottom: Spatial distribution of their antisymmetric combination $(|A S\rangle)$, living on the $B$ sublattice, in the same system. The axial magnetic field is set to be uniform here; i.e., $\chi(\mathcal{R})=q \mathcal{R}^{2}$, with $q=0.02$. 
A number of recent experiments have already revealed the existence of a zero-energy subband in strained real $[28,29]$ and artificial [30] graphene. In particular, when the axial (uniform) magnetic field $b \sim 60 \mathrm{~T}$ is introduced in strained molecular or artificial graphene, site-resolved STM measurements clearly indicate the existence of zeroenergy states on the $A$ sublattice in the interior of the system [30]. A careful examination, however, also shows that there are finite number of zero-energy states in the close vicinity of the system's boundary, which, on the other hand, are localized on the other sublattice $(B)$. In addition, the zero modes residing near the boundary are predominantly localized around three of the edges of the hexagonal strained molecular honeycomb lattice, connected through rotations by $2 \pi / 3$. All of these observations are in accordance with the spatial and sublattice structure of the zero modes, shown in Fig. 2, obtained by applying a specific modulation of the nearest-neighbor hopping amplitude (Fig. 1) that serves to capture the effect of the axial magnetic field in a finite honeycomb lattice. When $b \leq 45 \mathrm{~T}$, it becomes more difficult to discern the zero modes on sublattice $B$, although near the edge zerobias STM shows bright spots along the nearest-neighbor bonds, possibly revealing the overlap among the zeroenergy states localized on $A$ and $B$ sublattices. Our numerical analysis of the zero modes also exhibits the existence of such a region, where the zero modes on two sublattices overlap; see Fig. 2.

It is worth contrasting the structure of the zero-energy subspaces in the presence of true versus axial magnetic field. Coupling of Dirac fermions to the true magnetic field involves the same Eq. (1), except for the replacement of the matrix $i \gamma_{3} \gamma_{5}$ by the four-dimensional unit matrix and taking the matrix $M(\chi)$ to be $\exp \left[\left(\sigma_{0} \otimes i \gamma_{3} \gamma_{5}\right) \chi(\vec{x})\right]$. Likewise, the zero-energy states in the true magnetic field still have the form of $\Psi_{0, n}$ in Eq. (2), but with the matrix $\gamma_{0}$ in the exponent replaced by $i \gamma_{3} \gamma_{5}$. As a consequence, the normalizable zero modes now reside on both sublattices. In a finite graphene system in a true magnetic field, the near-zeroenergy states, residing in the bulk, similarly equally populate both sublattices. The non-normalizable zero modes in the continuum, located near the boundary in a finite system, are also shared equally between the two sublattices. Hence, in stark contrast to the axial field, in the presence of true magnetic field, the interior and the boundary of a finite honeycomb lattice have the same unresolved sublattice structure.

\section{MAGNETIC GROUND STATES WITH HUBBARD INTERACTION}

Next, we focus on the effect of electron-electron interaction in strained graphene, with the chemical potential $(\mu)$ tuned to the charge-neutrality point. Here, we consider only the on-site Hubbard interaction $(U)$ among the fermions. The standard Hubbard Hamiltonian is

$$
H=-t \sum_{\langle i, j\rangle, \sigma}\left(u_{i, \sigma}^{\dagger} v_{j, \sigma}+\text { H.c. }\right)+H_{U} \text {, }
$$

with the interaction Hamiltonian given by

$$
H_{U}=U \sum_{i}\left(n_{i, \uparrow}-\frac{1}{2}\right)\left(n_{i, \downarrow}-\frac{1}{2}\right)-\mu N,
$$

and where $n_{i, \sigma}$ is the fermionic number operator with spin projection $\sigma=\uparrow, \downarrow$ at site $i$. The total number of electrons in the system is $N$. The charge neutrality in the system is maintained through the constraint $\mu=0$. The usual Hartree decomposition of the on-site interaction leads to an effective single-particle Hamiltonian

$$
\begin{aligned}
H_{U}= & U \sum_{x=A, B}\left(\left\langle n_{x, \uparrow}\right\rangle-\frac{1}{2}\right)\left(n_{x, \downarrow}-\frac{1}{2}\right)+\left(\left\langle n_{x, \downarrow}\right\rangle-\frac{1}{2}\right) \\
& \times\left(n_{x, \uparrow}-\frac{1}{2}\right)-\mu N
\end{aligned}
$$

One can rewrite $\left\langle n_{x, \sigma}\right\rangle$ as

$$
\left\langle n_{A, \sigma}\right\rangle=1 / 2+\sigma \delta_{A, \sigma}(r), \quad\left\langle n_{B, \sigma}\right\rangle=1 / 2-\sigma \delta_{B, \sigma}(r),
$$

where $\sigma=(+,-)$, respectively, represents $(\uparrow, \downarrow)$ projections of the electron's spin along the $z$ axis. $\delta_{x, \sigma}(r)$ corresponds to the site-dependent local deviation of the electronic density from the uniform background, and is to be determined self-consistently in a finite honeycomb lattice in the presence of the axial magnetic field representing strain. Here, we always take the system to be quasicircular, and $r=1,2, \ldots, n$ is a discrete variable, representing the $n$th ring around the center of the system. In the presence of ordering $(\delta \neq 0)$, the overall charge neutrality of the system is achieved through the constraint

$$
\sum_{r} \sum_{\sigma= \pm}\left[\sigma \delta_{A, \sigma}(r)-\sigma \delta_{B, \sigma}(r)\right]=0
$$

in addition to $\mu=0$. Notice that the Fock decoupling of the Hubbard term would yield expectation values of the components of the local magnetization that are orthogonal to the chosen $(z)$ direction, which we neglect. Depending on the relative signs of the site parameters $\delta$, one can realize two different magnetic ground states: (i) If the ground state configuration is such that all $\delta_{A / B, \uparrow / \downarrow}>0$ in the above equation, then this would correspond to an antiferromagnetic phase, whereas (ii) a ground state with $\delta_{A, \uparrow / \downarrow}>0$, but $\delta_{B, \uparrow / \downarrow}<0$, would be identified as a ferromagnetic state. Before we proceed with the numerical simulation of the Hubbard model, it is worth pausing to compare these two magnetic phases in strained graphene. 
Magnetization on the two triangular sublattices points in opposite directions when the ground state is antiferromagnetic, whereas in a ferromagnet the two sublattice magnetizations are aligned. Recall that all of the zero modes in strained graphene are localized on one sublattice in the bulk and on the other sublattice near the boundary of a finite system. Therefore, both the Néel and the ferromagnetic orders in strained graphene give rise to a finite local magnetization everywhere in the system. However, globally the two states may be distinguished.

Because of the spatial separation of the zero modes that are localized on different sublattices, the magnetization in the state with an equal sign of the Néel order parameter in the entire system would need to change sign as one approaches the boundary from the bulk, so that the total magnetization could, in fact, vanish. In the truly ferromagnetic state, on the other hand, the sign of the magnetization in the bulk and the boundary would be the same, so that the system would develop an overall finite magnetization. We show, through a detailed numerical calculation, that by taking into account the entire zero subspace in a finite system, the magnetic ground state in strained graphene is uniquely determined to be of the first variety, i.e., a global antiferromagnet with zero total magnetization. Nevertheless, the continuum picture already provides valuable insight into the nature of the competition between the Néel and the ferromagnetic states. The order parameters of these two states read as $\vec{N}=\left\langle\Psi^{\dagger}\left(\vec{\sigma} \otimes \gamma_{0}\right) \Psi\right\rangle$ and $\vec{F}=\left\langle\Psi^{\dagger}\left(\vec{\sigma} \otimes I_{4}\right) \Psi\right\rangle$, respectively. Both states split the zero-energy subspace in strained graphene, and open a gap at the Dirac points. However, the matrix appearing in the Néel order parameter anticommutes with the Dirac Hamiltonian, and as such it corresponds to a chiral-symmetry-breaking mass term for the Dirac quasiparticles. In contrast, in the ferromagnetic order parameter, the corresponding matrix commutes with the Dirac Hamiltonian. As a result, in addition to the splitting of the zero-energy subspace common to both orders, the Néel order at the mean-field level pushes all of the filled states down in energy below the chemical potential. In contrast, the ferromagnetic order parameter splits all of the energy levels equally, half up and half down in energy, and, therefore, lowers only the energy of the halffilled zero-energy subspace. Thus, by spontaneously developing the Néel order, the system can minimize the ground state energy more efficiently.

\section{SELF-CONSISTENT CALCULATION}

Now, we present the results of the self-consistent calculation of the magnetic order parameters with on-site Hubbard interaction $(U)$ in strained graphene. We numerically search for the self-consistent solution of $\delta$ with two different initial Ansätze for the magnetic ordering (i) when $\delta_{A / B, \uparrow / \downarrow}>0$, which can be identified as antiferromagnet ordering, and (ii) $\delta_{A, \uparrow / \downarrow}>0, \delta_{B, \uparrow / \downarrow}<0$, which corresponds to a ferromagnetic order. Here, all of the $\delta$ are kept as a function of position, and we always maintain the overall charge neutrality of the system. In the Hartree selfconsistent calculation, electronic spin is treated as an Ising variable, $\vec{\sigma} \rightarrow \sigma_{3}$, and the effect of fluctuation is neglected for a moment. Later, we treat the fermionic spin as $O(3)$ vectors in a separate quantum Monte Carlo simulation of the Hubbard model in strained graphene, which explicitly takes into account the effect of the fluctuations. Here, we search for the self-consistent solution of the magnetic order specifically for weak Hubbard interactions, $U \ll U_{c}$, where $U_{c} \approx 3.8 t$ is the zero axial field critical strength of the on-site interaction for antiferromagnetic ordering [16]. Because of the presence of a finite density of state near the zero energy in strained graphene, ordering takes place even for on-site interaction as weak as $U / t=0.1$, irrespective of the spatial profile of the axial magnetic field, and a spectral gap opens up at the Dirac points. The resulting magnetic ground state is insensitive to the initial Ansatz (i) or (ii), and thus our self-consistent analysis can be considered as variational calculation. To further explore the nature of this magnetic ground state, we define two local order parameters as

$$
\begin{aligned}
& N(r)=\frac{1}{2}\left(\delta_{A, \uparrow}+\delta_{A, \downarrow}+\delta_{B, \uparrow}+\delta_{B, \downarrow}\right)(r), \\
& M(r)=\frac{1}{2}\left(\delta_{A, \uparrow}+\delta_{A, \downarrow}-\delta_{B, \uparrow}-\delta_{B, \downarrow}\right)(r),
\end{aligned}
$$

which correspond to local Néel and ferromagnetic order parameters, respectively. $\delta$ are as defined in Eq. (7).

Here, we obtain the numerical results in a quasicircular honeycomb lattice of 600 sites or $r_{\max }=10$. In such a system, the self-consistent solutions for all the $\delta$ are essentially without any finite size effects, for all values of the subcritical Hubbard interactions, down to $U=0.1$, and for both the uniform and localized axial fields (see the captions of Figs. 3 and 4 for details of these parameters). Self-consistent solutions of $N(r)$ and $M(r)$ in the presence of roughly uniform axial magnetic fields and a wide range of subcritical on-site interactions are shown in the upper and lower panels of Fig. 3, respectively. From the spatial variation of these two order parameters, we see that the local antiferromagnetic order parameter $N(r)$ is of the same sign in the bulk as well as in the boundary of the system, whereas $M(r)$ near the boundary is of the opposite sign from the bulk. The total magnetization for all chosen values of the interaction and uniform axial fields is zero within the numerical accuracy $\sim 10^{-12}$. Therefore, the magnetic ground state is indeed an antiferromagnet, as one would anticipate from the continuum description of this problem. The Néel order in strained graphene is different from the conventional one on the honeycomb lattice [16], as it also carries a finite local magnetization everywhere in the system. We call this unconventional magnetic ground state a global (edge-compensated) antiferromagnet.

We also obtain the self-consistent solution for the magnetic ground state when the graphene flake is subject to 

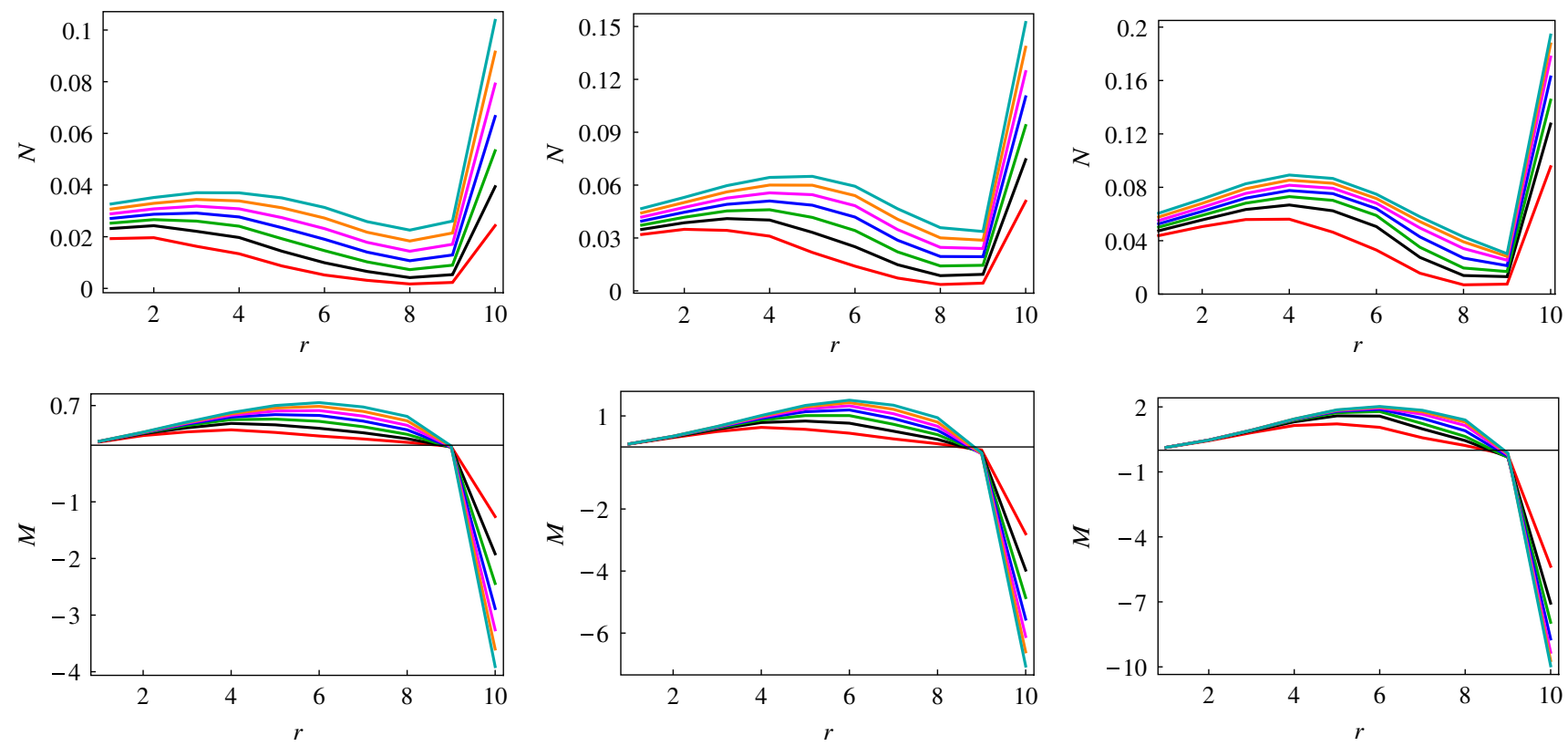

FIG. 3. Upper panels: Spatial variation of the local Néel order parameter $(N)$, defined in Eq. (9), obtained from the self-consistent solution of the magnetic ground state, for various subcritical on-site Hubbard interaction and roughly uniform axial magnetic fields. The strength of the Hubbard interaction in each plot reads as $U=0.1$ (red line), 0.2 (black line), 0.3 (green line), 0.4 (blue line), 0.5 (magenta line), 0.6 (orange line), 0.7 (cyan line) from bottom to top. Strength of the uniform axial field reads as $b=0.025 b_{0}, 0.035 b_{0}, 0.045 b_{0}$ from left to right, where $b_{0}=\hbar /\left(e a^{2}\right) \sim 10^{4} \mathrm{~T}$ is the axial field associated with the lattice spacing $a \approx 2.5 \AA$. Lower panels: Spatial variation of local ferromagnetic order parameter $(M)$ obtained from the same self-consistent solution for the magnetic ground state. Here, $N$ is the average Néel order parameter per unit cell, and $M$ is the total magnetization, in a quasicircular ring.
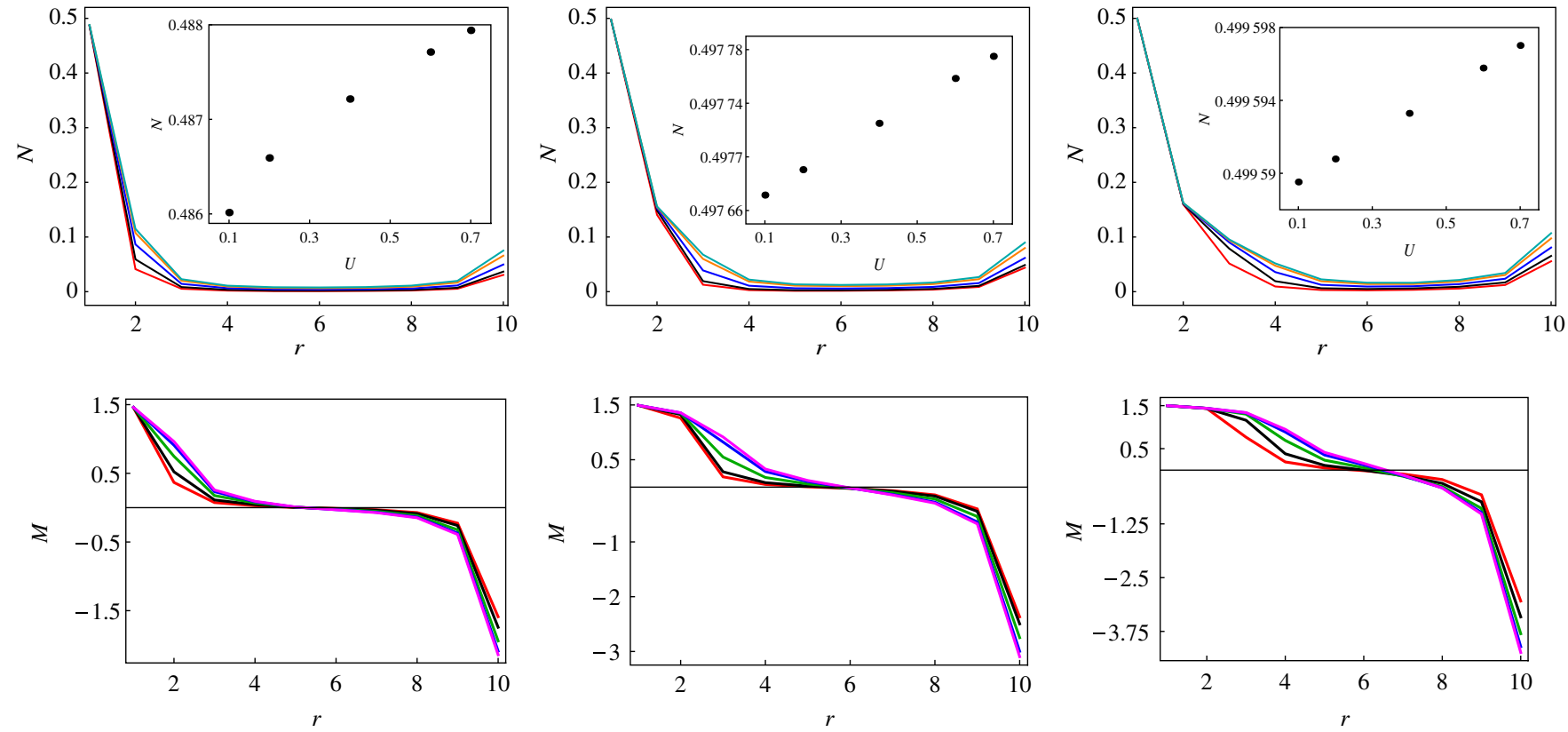

FIG. 4. Upper panels: Spatial variation of the local Néel $(N)$ order parameter, obtained from the self-consistent solution of the magnetic ground state, as in Eq. (9), for various subcritical Hubbard interactions, and bell-shaped localized axial flux peaked around the center of the system. The strength of the Hubbard interaction in each plot reads as $U=0.1$ (red line), 0.2 (black line), 0.4 (blue line), 0.6 (orange line), 0.7 (dark green line) from bottom to top. Total flux of the localized axial field reads as $\Phi_{\text {total }}=7.85 \Phi_{0}, 9.42 \Phi_{0}, 10.99 \Phi_{0}$ from left to right, where $\Phi_{0}$ is the flux quanta. Inset in upper panels: Variation of $N(r)$ at the center of the system with the strength of the on-site interaction. Lower panels: Spatial variation of local ferromagnet order $(M)$ in the same magnetic ground state for $U=0.1$ (red line), 0.2 (black line), 0.4 (dark green line), 0.6 (blue line), 0.7 (magenta line). Total flux of the localized axial field in the lower panel is same as in upper one. Here, $N$ and $M$ are the same as in Fig. 3. 
nonuniform axial fields, localized in the vicinity of the center of the system. The spatial variation of the local order parameters $N(r)$ and $M(r)$, for a wide range of subcritical interactions, and the total flux of the localized axial magnetic field are shown in the upper and lower panels of Fig. 4, respectively, and were obtained in a quasicircular system of 600 sites $\left(r_{\max }=10\right)$. From Figs. 3 and 4, it is clear that the nature of the magnetic ground state is insensitive to the exact profile of the axial field, and it is always the global antiferromagnet. However, the location where the local magnetization changes its sign depends on the profile of the axial field. In the presence of a uniform axial field, magnetization flips its sign only very close to the boundary of the system, whereas in the presence of localized fields the same change occurs in the middle of the system. The difference in the position of the domain wall between the two signs of the magnetization is tied to the spatial distribution of the zero-energy states in the system. In the uniform axial field, the zero modes on the $A$ sublattice are distributed over a large portion of the bulk, whereas they are highly localized in the deep interior when the axial field is peaked near the center of the system. Therefore, the local magnetization suffers a change in sign roughly where the zero-energy states on the $A$ sublattice lose their support. The magnitude of both of the order parameters $N(r)$ and $M(r)$ increases with the strength of the interaction and of the axial field, as shown in Figs. 2 and 3.

The spatial distribution of the zero modes also dictates spatial variation of the Néel order parameter. Note that, in the presence of uniform axial fields, the Néel order parameter $N(r)$ is more or less uniform in the bulk $(r \leq 6)$ of the system (see Fig. 3, upper panel). The abrupt increment in $N(r)$ near the boundary of the system arises from the existence of zero-energy states on the $B$ sublattice in that region. When the axial field assumes a spatially localized profile, around the center of the system, the Néel order parameter predominantly develops in the region where the axial flux penetrates the graphene flake; see Fig. 4 (upper panel). The existence of the zero-energy states

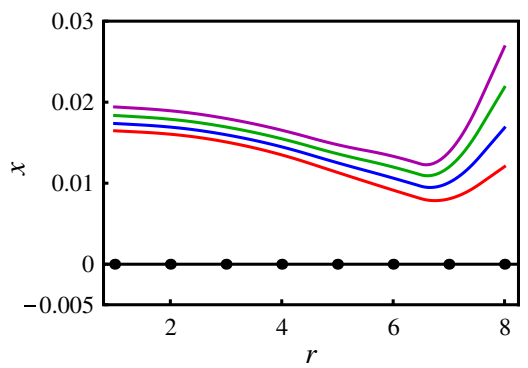

on the sites of the $B$ sublattice near the boundary leads to the spikes in $N(r)$ even when the axial field is inhomogeneous. Therefore, the spatial modulation of the Néel order closely follows the profile of the axial magnetic field, resembling, in this regard, the spatial variation of the quantum anomalous Hall insulator in strained graphene with next-nearest-neighbor interaction [24].

\section{HUBBARD MODEL IN TRUE MAGNETIC FIELD}

The conical dispersion of the Dirac quasiparticles quenches into a set of well separated Landau levels, when the graphene flake is subject to a uniform true magnetic field. Although the discrete quantization of the spectrum smears out if the magnetic field is spatially modulated, a finite number of states at zero-energy states always persists, irrespective of the profile of the magnetic fields [27]. Finite density of states near the Dirac points, here as well, catalyzes the effect of electron-electron interactions. To compare the present unconventional magnetic ground state of the Hubbard model in strained graphene with the one in the presence of a true magnetic field, we perform the same numerical self-consistent analysis in a finite honeycomb lattice, placed in uniform and nonuniform true magnetic fields. The orbital effect of the true magnetic field is included by incorporating the Peierls phase into the nearest-neighbor hopping amplitudes [36]. Here, we omit the single-particle Zeeman coupling of the electron's spin with the magnetic field $[37,38]$. In true magnetic field the zero-energy states are found on both sublattices in the bulk, as well as near the boundary of the system. In this situation, only the antiferromagnetic Ansatz (i) leads to a finite gap at the Dirac point.

From the self-consistent solution of the magnetic ground state, obtained in a finite honeycomb system of 384 sites $\left(r_{\max }=8\right)$, we compute the two local order parameters $N(r)$ and $M(r)$, defined in Eq. (9). The results are presented in Fig. 5, when the field is uniform (left) and nonuniform

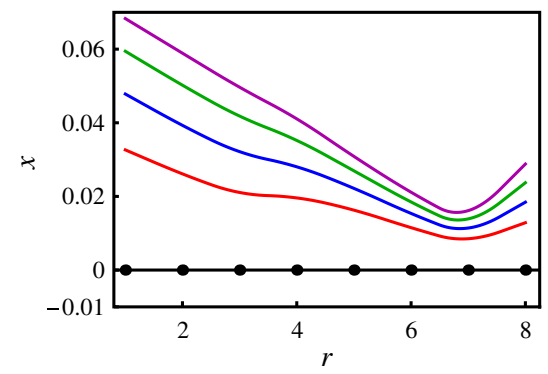

FIG. 5. Left: Spatial variation of the Néel (solid lines, with $x=N$ ) and ferromagnetic (black dots, with $x=M$ ) order parameters per unit cell, in the presence of uniform true magnetic field of strength $250 \mathrm{~T}$. Right: The same quantities in a localized true magnetic field, of the strength $650 \mathrm{~T}$ at the center of the system. The average magnetic field at each quasicircular ring of the honeycomb lattice decreases as $B(r)=(690-40 r) \mathrm{T}$, towards the boundary of the system. Strength of the Hubbard interaction reads as $U=0.4$ (red line), 0.5 (blue line), 0.6 (dark green line), 0.7 (purple line). The ferromagnetic order is identically zero for all values of $U$, both in uniform and nonuniform true magnetic fields. 
(right) (once again, a bell-shaped one, peaked around the center of the system). The local Néel order parameter $N(r)$ again follows the profile of the true magnetic field, resembling, in this regard, the spatial distribution of the charge-density-wave order, obtained previously for spinless fermions in a honeycomb lattice with the nearest-neighbor interaction [36]. Because of the existence of a finite density of states at the Dirac point, the antiferromagnetic ordering can be found for the interaction as weak as $U=0.4$ [39]. On the other hand, $M(r)$ is zero everywhere in the system. The dramatic difference in the magnetic ground states between the axial and the true magnetic fields arises entirely as a consequence of the distinct structure of the zero modes. The antiferromagnetic ground state we find in the presence of a true magnetic field and at weak interaction $\left(U \ll U_{c}\right)$ is the exact replica of the one in graphene at strong interaction $(U \geq 3.8 t)$ and in zero field.

\section{MONTE CARLO CALCULATION}

Axial magnetic fields, resulting from the modification of the hopping matrix elements between the nearest neighbors according to Eq. (3), do not break the particle-hole symmetry. Therefore, auxiliary field quantum Monte Carlo simulations do not suffer from the infamous minus sign problem and accurate simulations on large lattices can be carried out. Here, we use the projective zero temperature approach based on the equation

$$
\frac{\left\langle\Psi_{0}|O| \Psi_{0}\right\rangle}{\left\langle\Psi_{0} \mid \Psi_{0}\right\rangle}=\lim _{\Theta \rightarrow \infty} \frac{\left\langle\Psi_{T}\left|e^{-\Theta H} O e^{-\Theta H}\right| \Psi_{T}\right\rangle}{\left\langle\Psi_{T}\left|e^{-2 \Theta H}\right| \Psi_{T}\right\rangle},
$$

in which the ground state is filtered out of a single Slater determinant by propagating along the imaginary time axis. It is beyond the scope of this paper to go into the details of the implementation, and the readers are referred to Ref. [40] for an overview of the algorithm. Let us, however, comment on some aspects of our implementation. The axial field does not break SU(2)-spin rotation symmetry, and we find it important to impose this symmetry by opting for a discrete Hubbard-Stratonovitch transformation coupling to the charge:

$$
e^{-\Delta \tau U\left(n_{i}-1\right)^{2}}=\frac{1}{2} \sum_{s= \pm 1} e^{i \alpha s\left(n_{i}-1\right)},
$$

with $\cos (\alpha)=e^{-\Delta \tau U / 2}$. Furthermore, we use a symmetric Trotter decomposition with $\Delta \tau t=0.1$, and the trial wave function corresponds to the ground state of the noninteracting Hamiltonian. For this choice of the trial wave function, projection parameters in the range $\Theta t=40-60$ are sufficient to guarantee convergence to the ground state within the quoted accuracy.

Since the Monte Carlo simulations do not break SU(2) spin symmetry, we have to rely on spin-spin correlations to detect the global, or edge-compensated, antiferromagnetic state. In Fig. 6 (upper panel), we consider 600-site flakes at $U / t=2$. This choice of $U / t$ places us well below $U_{c} \approx 3.8 t$, at which the transition to the antiferromagnetic Mott insulating state occurs in the absence of axial field [16]. The reference site $\boldsymbol{i}_{\mathrm{R}}=(-1,-1 / \sqrt{3}) / 2$ is chosen to belong to the sublattice, which hosts the (normalizable) zero-energy modes. In very close vicinity to $\boldsymbol{i}_{\mathrm{R}}$, antiferromagnetic correlations are apparent, and they rapidly give way to dominant ferromagnetic correlations. For the uniform axial field, $\chi(\mathcal{R})=q \mathcal{R}^{2}$, the extent of the dominant ferromagnetic correlations is considerably larger than for the localized one, $\chi(\mathcal{R})=q \log (\mathcal{R})$. Finally, strong antiferromagnetic correlations are present at the edge of the flake. Hence, the overall features present in the quantum Monte Carlo calculations support the mean-field picture of the global, or edge-compensated, antiferromagnetic spin structure.

A more precise measure for the global edgecompensated antiferromagnetic state can be obtained by considering

$$
S(R)=\sum_{|j|<R}\left\langle S_{i_{\mathrm{R}}} \cdot \boldsymbol{S}_{i_{\mathrm{R}}+j}\right\rangle .
$$

Figure 6 (lower panel) plots this quantity for the uniform and localized axial fields and at various values of $q$. Owing to the singlet nature of the ground state on finite lattices, $S(R)=0$, when $R$ exceeds the radius of the flake. In the absence of axial field $(q=0)$, only short-range antiferromagnetic correlations are present and $S(R)$ quickly decays to zero. At finite values of the axial field, $S(R)$ images the global edge-compensated antiferromagnetic spin structure. For the localized field, the zero-energy modes are localized around the center and $S(R)$ quickly approaches a plateau value before being compensated by the edge antiferromagnetic correlations. In contrast, for the uniform field, $S(R)$ builds up as a function of distance before again being compensated by the edge magnetism.

In Fig. 7, we consider larger values of $U / t=4$. In the absence of the axial field, this choice of the Hubbard interaction places us in the antiferromagnetic Mott insulting phase, shown through the spin-spin correlation in Fig. 7 (top left). As a consequence, and in comparison to the $U / t=2$ case, the integrated spin-spin correlations of Eq. (12) show small fluctuations up to large distances. It is interesting to note that, even starting from the Mott insulating state, the axial field leads to the same reorganization of the spin-spin correlations as observed at weak couplings, shown in Fig. 7 (bottom). One will, nevertheless, observe substantial antiferromagnetic oscillations superimposed on the edge-compensated antiferromagnetic spin structure. The strain-induced restructure of the spin-spin correlation for supercritical interactions $(U / t=4)$ is shown in Fig. 7 (top) for roughly uniform (middle) and localized (right) axial fields. 

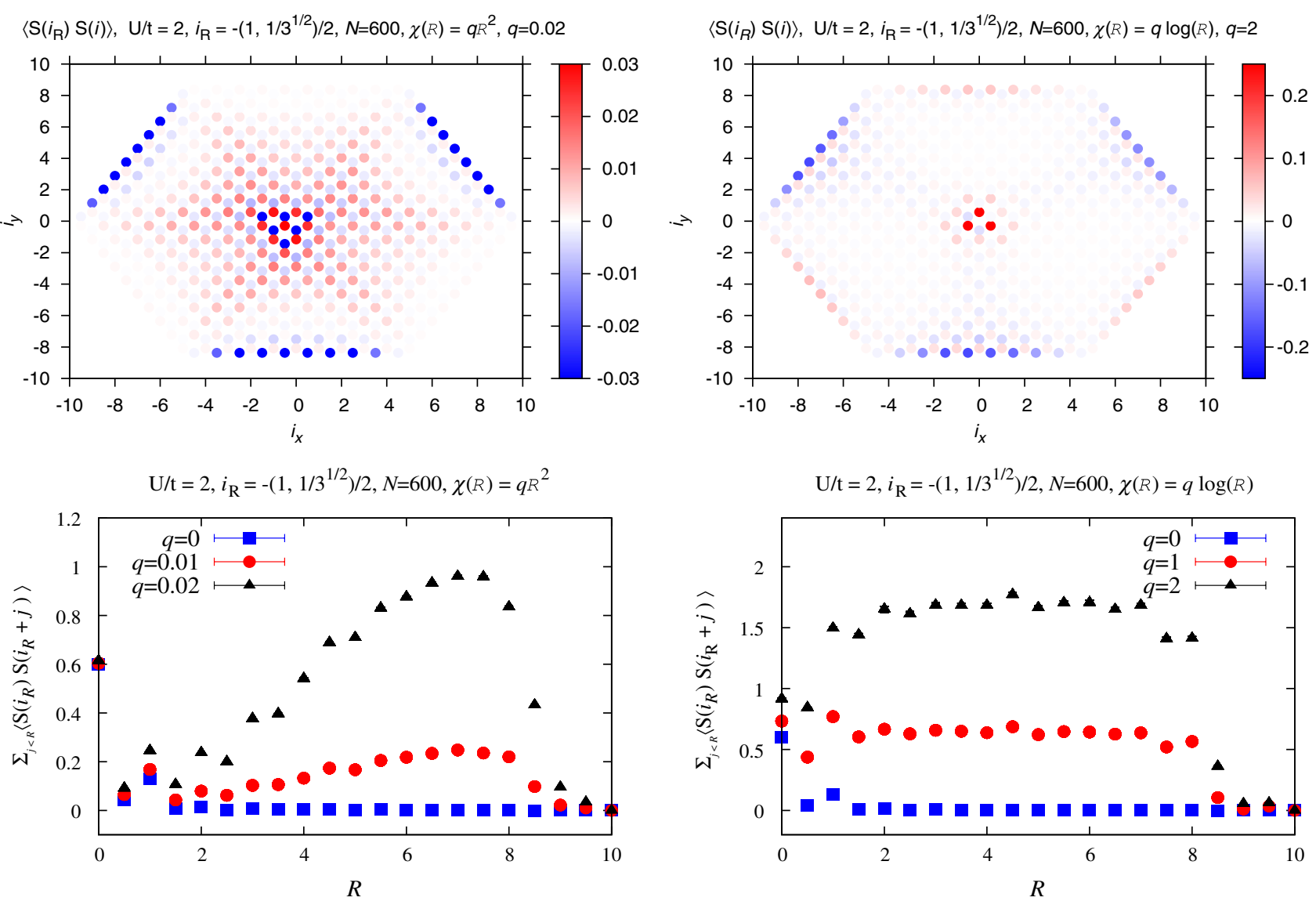

FIG. 6. Quantum Monte Carlo simulations on a 600-site flake. The top panels plot the spin-spin correlation functions between the reference point $\boldsymbol{i}_{R}=(-1 / 2,-1 / 2 \sqrt{3})$ and other sites of the lattice for both the uniform $\chi(\mathcal{R})=q \mathcal{R}^{2}$ and $\operatorname{local} \chi(\mathcal{R})=q \log (\mathcal{R})$ axial fields. The two bottom panels plot the integrated spin-spin correlations $S(R)$, defined in Eq. (12), exhibiting, at finite values of $q$, edgecompensated antiferromagnetic spin structure.

\section{SUMMARY AND DISCUSSION}

To summarize, we propose a specific modulation of the nearest-neighbor hopping amplitudes in honeycomb lattice that captures the coupling of the low-energy Dirac quasiparticles to the (time-reversal-symmetric) axial magnetic fields. Because of the presence of the axial magnetic field, a finite number of states appears at (near) zero energy, which in turn enhances the effect of electron-electron interaction. Various orderings can take place this way in strained graphene even at weak interactions [22-25].

In this paper, we consider only the on-site Hubbard interaction between the fermions and study the nature of the magnetic ground state in strained graphene. Because of the special structure of the zero-energy states, which are supported by one sublattice in the bulk of the system and by the other one near the boundary, the magnetic ground state in strained graphene lacks any analog in pristine graphene, or in graphene in true magnetic fields. Through the numerical self-consistent Hartree calculation, and a separate quantum Monte Carlo simulation, we establish that the magnetic ground state gives rise to both antiferromagnetic and ferromagnetic orders, locally, everywhere in the system. Although the antiferromagnetic order parameter is of the same sign in the entire system, the magnetization changes its sign near the boundary, so that the total magnetization is actually zero. Such ordering takes place even for weak Hubbard interactions. We call the magnetic ground state in strained graphene edgecompensated, or global, antiferromagnet.

In contrast, the ground state of the Hubbard model on a honeycomb lattice subject to true magnetic fields (and with the Zeeman coupling ignored $[37,38]$ ) is the conventional Néel antiferromagnet. Through self-consistent calculation, we show that such Néel ordering takes place again for weak interactions, and the order parameter closely resembles the profile of the true magnetic fields. In this case, however, the magnetization is identically zero everywhere in the system.

The experimental detection of the global antiferromagnetic phase relies on the measurement of local magnetic moment everywhere in the system, as well as on the existence of the zero-energy states, particularly near the edge of the system. Recent STM measurements in strained molecular graphene indicate the appearance of zero modes in the bulk and close to the boundary of the system, which are indeed 

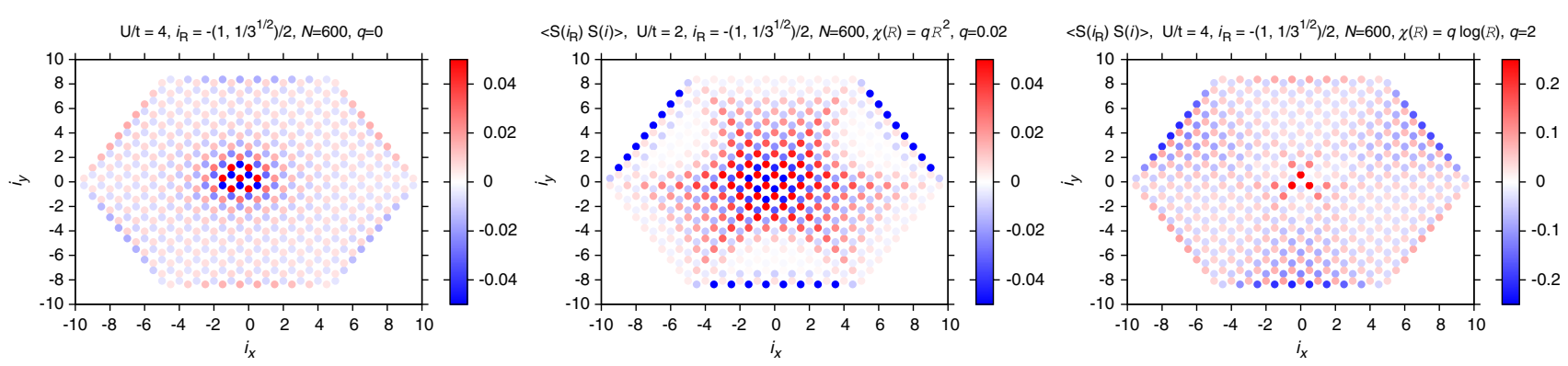

$\mathrm{U} / \mathrm{t}=4, i_{\mathrm{R}}=-\left(1,1 / 3^{1 / 2}\right) / 2, N=600, \chi(R)=q R^{2}$
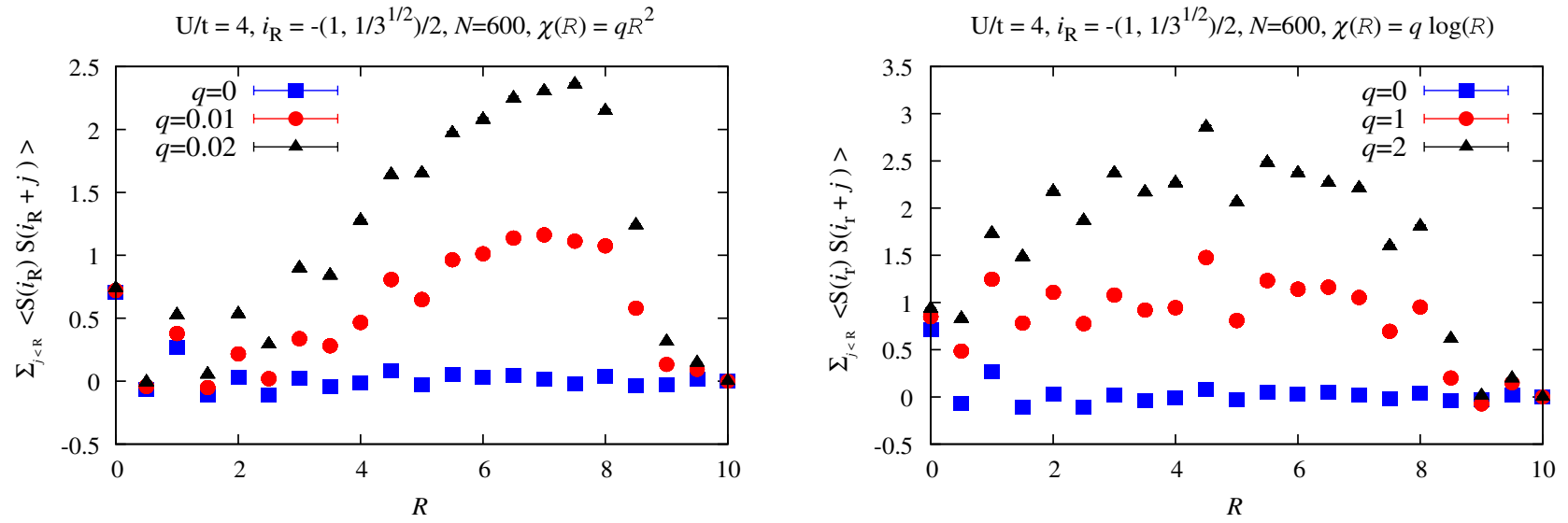

FIG. 7. Quantum Monte Carlo simulations on a 600-site flake. Top panels: Spin-spin correlation functions between the reference point $\boldsymbol{i}_{\mathrm{R}}=(-1 / 2,-1 / 2 \sqrt{3})$ and other sites of the lattice in the absence of axial field $[\chi(\mathcal{R})=0]$ (left), and in the presence of uniform $\left[\chi(\mathcal{R})=q \mathcal{R}^{2}\right]$ (middle) and local $[\chi(\mathcal{R})=q \log (\mathcal{R})]$ (right) axial fields. Bottom panels: Integrated spin-spin correlations $S(R)$ [see Eq. (12)] at $U / t=4$ for the uniform $\chi(\mathcal{R})=q \mathcal{R}^{2}$ (left) and local $\chi(\mathcal{R})=q \log (\mathcal{R}$ ) (right) axial fields.

living on two different sublattices [30], in agreement with our numerical analysis (see Fig. 2). The local magnetic moment, on the other hand, can be probed by either magnetic force microscope or spin-polarized STM measurement. The latter method successfully establishes an antiferromagnetic ordering on monolayer Fe, resting on tungsten [41], and reveals the spin structure inside a magnetic vortex core [42]. A systematic measurement of the local magnetic moment using the spin-polarized STM may, therefore, also detect the unconventional magnetic ground state in strained graphene. The desired suppression of the real-time fluctuations of the local magnetic moment can be obtained either by increasing the system's size or by increasing the size of the magnetic moments, which, as our analysis suggests, can be achieved by enhancing the strength of the axial magnetic field and/or the strength of the on-site interaction. The latter can possibly be tuned to a certain degree in the molecular or artificial graphene. Since the local ferromagnetic moment changes its sign roughly where the zero modes lose their support on the $A$ sublattice, it is perhaps comparatively easier to detect the proposed global antiferromagnetic phase in strained graphene, when the axial magnetic field is localized near the center of the system. In that situation, the ferromagnetic domain wall appears somewhere in the middle of the system (see Fig. 4), whereas, on the other hand, it lies only very close to the boundary when the axial field is uniform (see Fig. 3).

\section{ACKNOWLEDGMENTS}

B. R. was supported at National High Magnetic Field Laboratory by NSF cooperative agreement No. DMR0654118, the State of Florida, and the U.S. Department of Energy. F. F. A. is supported by the DFG, under Grants No. AS120/9-1 and No. AS120/10-1 (Forschergruppe FOR 1807). I.F. H. has been supported by the NSERC of Canada. We thank the Jülich Supercomputing Centre and the Leibniz-Rechenzentrum in Münich for generous allocation of CPU time.

[1] J. González, F. Guinea, and M. A. H. Vozmediano, Marginal-Fermi-Liquid Behavior from Two-Dimensional Coulomb Interaction, Phys. Rev. B 59, R2474 (1999).

[2] S. Sorella and E. Tosatti, Semi-Metal-Insulator Transition of the Hubbard Model in the Honeycomb Lattice, Europhys. Lett. 19, 699 (1992).

[3] T. Paiva, R. T. Scalettar, W. Zheng, R. R. P. Singh, and J. Oitmaa, Ground-State and Finite-Temperature Signatures of Quantum Phase Transitions in the Half-Filled Hubbard Model on a Honeycomb Lattice, Phys. Rev. B 72, 085123 (2005).

[4] D. V. Khveshchenko, Ghost Excitonic Insulator Transition in Layered Graphite, Phys. Rev. Lett. 87, 246802 (2001). 
[5] D. Khveshchenko and H. Leal, Excitonic Instability in Layered Degenerate Semimetals, Nucl. Phys. B687, 323 (2004).

[6] I. F. Herbut, Interactions and Phase Transitions on Graphene's Honeycomb Lattice, Phys. Rev. Lett. 97, 146401 (2006).

[7] I. F. Herbut, V. Juričić, and B. Roy, Theory of Interacting Electrons on the Honeycomb Lattice, Phys. Rev. B 79, 085116 (2009).

[8] J. E. Drut and T. A. Lähde, Is Graphene in Vacuum an Insulator?, Phys. Rev. Lett. 102, 026802 (2009).

[9] J. E. Drut and T. A. Lähde, Lattice Field Theory Simulations of Graphene, Phys. Rev. B 79, 165425 (2009).

[10] J.E. Drut and T. A. Lähde, Critical Exponents of the Semimetal-Insulator Transition in Graphene: A Monte Carlo Study, Phys. Rev. B 79, 241405 (2009).

[11] E. V. Castro, A. G. Grushin, B. Valenzuela, M. A. H. Vozmediano, A. Cortijo, and F. de Juan, Topological Fermi Liquids from Coulomb Interactions in the Doped Honeycomb Lattice, Phys. Rev. Lett. 107, 106402 (2011).

[12] A. G. Grushin, E. V. Castro, A. Cortijo, F. de Juan, M. A. H. Vozmediano, and B. Valenzuela, Charge Instabilities and Topological Phases in the Extended Hubbard Model on the Honeycomb Lattice with Enlarged Unit Cell, Phys. Rev. B 87, 085136 (2013).

[13] J. González, Dynamical Breakdown of Parity and TimeReversal Invariance in the Many-Body Theory of Graphene, J. High Energy Phys. 07 (2013) 175.

[14] Z. Y. Meng, T. C. Lang, S. Wessel, F. F. Assaad, and A. Muramatsu, Quantum Spin Liquid Emerging in Two-Dimensional Correlated Dirac Fermions, Nature (London) 464, 847 (2010).

[15] S. Sorella, Y. Otsuka, and S. Yunoki, Absence of a Spin Liquid Phase in the Hubbard Model on the Honeycomb Lattice, Sci. Rep. 2, 992 (2012).

[16] F. F. Assaad and I. F. Herbut, Pinning the Order: The Nature of Quantum Criticality in the Hubbard Model on Honeycomb Lattice, Phys. Rev. X 3, 031010 (2013).

[17] T. C. Lang, Z. Y. Meng, A. Muramatsu, S. Wessel, and F. F. Assaad, Dimerized Solids and Resonating Plaquette Order in SU(N)-Dirac Fermions, Phys. Rev. Lett. 111, 066401 (2013).

[18] I. F. Herbut, V. Juričić, and O. Vafek, Relativistic Mott Criticality in Graphene, Phys. Rev. B 80, 075432 (2009).

[19] B. Roy, Multicritical Behavior of $Z_{2} \times O(2)$ Gross-NeveuYukawa Theory in Graphene, Phys. Rev. B 84, 113404 (2011).

[20] B. Rosenstein, H.-L. Yu, and A. Kovner, Critical Exponents of New Universality Classes, Phys. Lett. B 314, 381 (1993).

[21] V. N. Kotov, B. Uchoa, V. M. Pereira, F. Guinea, and A. H. Castro Neto, Electron-Electron Interactions in Graphene: Current Status and Perspectives, Rev. Mod. Phys. 84, 1067 (2012).

[22] I. F. Herbut, Pseudomagnetic Catalysis of the Time-Reversal Symmetry Breaking in Graphene, Phys. Rev. B 78, 205433 (2008).

[23] P. Ghaemi, J. Cayssol, D. N. Sheng, and A. Vishwanath, Fractional Topological Phases and Broken Time-Reversal Symmetry in Strained Graphene, Phys. Rev. Lett. 108, 266801 (2012).
[24] B. Roy and I. F. Herbut, Topological Insulators in Strained Graphene at Weak Interaction, Phys. Rev. B 88, 045425 (2013).

[25] B. Roy and V. Juričić, Strain-Induced Time-Reversal Odd Superconductivity in Graphene, arXiv:1309.0507.

[26] F. Guinea, M. I. Katsnelson, and A. K. Geim, Energy Gaps and a Zero-Field Quantum Hall Effect in Graphene by Strain Engineering, Nat. Phys. 6, 30 (2010).

[27] Y. Aharonov and A. Casher, Ground State of a Spin-1/2 Charged Particle in a Two-Dimensional Magnetic Field, Phys. Rev. A 19, 2461 (1979).

[28] N. Levy, S. A. Burke, K. L. Meaker, M. Panlasigui, A. Zettl, F. Guinea, A. H. C. Neto, and M. F. Crommie, Strain-Induced PseudoMagnetic Fields Greater Than 300 Tesla in Graphene Nanobubbles, Science 329, 544 (2010).

[29] J. Lu, A. H. C. Neto, and K. P. Loh, Transforming Moiré Blisters into Geometric Graphene Nano-bubbles, Nat. Commun. 3, 823 (2012).

[30] K. K. Gomes, W. Mar, W. Ko, F. Guinea, and H. C. Manoharan, Designer Dirac Fermions and Topological Phases in Molecular Graphene, Nature (London) 483, 306 (2012).

[31] R. Jackiw and S.-Y. Pi, Chiral Gauge Theory for Graphene, Phys. Rev. Lett. 98, 266402 (2007).

[32] B. Roy, Odd Integer Quantum Hall Effect in Graphene, Phys. Rev. B 84, 035458 (2011).

[33] B. Roy, Magnetic-Field Induced Inequivalent Vortex Zero Modes in Strained Graphene, Phys. Rev. B 85, 165453 (2012).

[34] B. Roy, Z.-X. Hu, and K. Yang, Theory of Unconventional Quantum Hall Effect in Strained Graphene, Phys. Rev. B 87, 121408 (2013).

[35] O. Motrunich, K. Damle, and D. A. Huse, Particle-Hole Symmetric Localization in Two Dimensions, Phys. Rev. B 65, 064206 (2002).

[36] B. Roy and I. F. Herbut, Inhomogeneous Magnetic Catalysis on Graphenes Honeycomb Lattice, Phys. Rev. B 83, 195422 (2011).

[37] I. F. Herbut, SO(3) Symmetry between Néel and Ferromagnetic Order Parameters for Graphene in a Magnetic Field, Phys. Rev. B 76, 085432 (2007).

[38] B. Roy, Theory of Integer Quantum Hall Effect in Insulating Bilayer Graphene, Phys. Rev. B 89, 201401(R) (2014).

[39] I. F. Herbut, Theory of Integer Quantum Hall Effect in Graphene, Phys. Rev. B 75, 165411 (2007).

[40] F. F. Assaad and H. G. Evertz, in Computational Many Particle Physics, Lecture Notes in Physics Vol. 739, edited by H. Fehske, R. Schneider, and A. Weiße (Springer-Verlag, Berlin, 2008), pp. 277-356.

[41] A. Kubetzka, P. Ferriani, M. Bode, S. Heinze, G. Bihlmayer, K. von Bergmann, O. Pietzsch, S. Blügel, and R. Wiesendanger, Revealing Antiferromagnetic Order of the Fe Monolayer on W(001): Spin-Polarized Scanning Tunneling Microscopy and First-Principles Calculations, Phys. Rev. Lett. 94, 087204 (2005).

[42] A. Wachowiak, J. Wiebe, M. Bode, O. Pietzsch, M. Morgenstern, and R. Wiesendanger, Direct Observation of Internal Spin Structure of Magnetic Vortex Cores, Science 298, 577 (2002). 